\title{
ON THE STRUCTURE OF PRINCIPAL IDEALS OF OPERATORS
}

\author{
BY
}

G. D. ALLEN AND L. C. SHEN

\begin{abstract}
This paper considers various types of principal ideals generated by single compact operators on a separable Hilbert space. In particular, necessary and sufficient condtions that a principal ideal be normable are given. Relations between principal ideals and duals of Lorentz and Orlicz spaces are also given. All conditions are expressed using the singular numbers of the operator.
\end{abstract}

1. Introduction. The objective of this paper is to examine certain aspects of the principal ideal structure of bounded linear operators, $\mathscr{B}(\mathcal{H C})$, on a separable Hilbert space. For example, we will give necessary and sufficient conditions that a principal ideal be normable. We shall exhibit a class of principal ideals equivalent to Orlicz spaces. We will also show a connection between certain principal ideals and the $\delta_{\Pi}$ classes of Gohberg and Krein.

Let $T \in \mathcal{K}(\mathcal{H})$, the compact operators on $\mathcal{H C}$, and let $\left\{s_{n}(T)\right\}$ be the eigenvalues of $\sqrt{T^{*} T}$, arranged in decreasing order and counted according to multiplicity. The values $s_{n}(T)$ are called the singular numbers of $T$. We denote by $\mathscr{P}(T)$ the principal ideal generated by $T$. We will show that there exists a class of principal ideals $\mathscr{P}(T)$ which are characterized by the set of operators $\Re$ for which $s_{n}(\Re)=O\left(s_{n}(T)\right)$. We shall also show that if $\mathscr{P}(T) \subsetneq \mathscr{P}(R)$, then there is an $S \in \mathcal{K}(\mathcal{H})$ such that $\mathscr{P}(T) \subsetneq \mathscr{P}(S) \subsetneq \mathscr{P}(R)$.

The principal tool used in this investigation is the theorem proved by Calkin [4] which asserts the existence of a one-to-one lattice serving correspondence between the two-sided ideals of $\mathscr{B}(\mathcal{F C})$ and sets of bounded sequences of positive numbers. This tool was improved by Morris and Salinas [6] to the extent that one need only consider sets of positive decreasing sequences. Recently, Blass and Weiss [2] and Weiss [11] have further refined this tool by defining a "frequency sequence" associated with each positive sequence.

In $\$ 2$ we consider types of regularity conditions on sequences which will form the basis of our results on ideals. Also we begin our discussion of the frequency method. In $\$ 3$ we give our fundamental structure theorems for

Received by the editors September 16, 1976.

AMS (MOS) subject classifications (1970). Primary 47B05, 47K05. 
principal ideals generated by operators having singular numbers satisfying the aforementioned regularity conditons. In $\$ 4$ we will further develop the frequency method by giving various applications of the Schweinsberg [10] theorem stating that every principal ideal $\mathscr{P}(T)$ is the intersection of all ideals

$$
\delta(f)=\left\{A \in \mathscr{B}(\mathcal{H}): \sum f\left(\alpha s_{n}(A)\right)<\infty, \text { for some } \alpha>0\right\},
$$

where $f$ is an admissible function and where $T \in \delta(f)$.

In the remainder of this section we introduce notation and give preliminary results used throughout this paper.

We first define the following classes of sequences of real numbers:

$c$ - the set of all bounded positive sequences;

$c_{0}$ - the set of all positive sequences which have limit zero;

$c_{0}^{+}-$the subset of $c_{0}$ of decreasing sequences;

$c_{p}$ - the subset of $l_{p}$ of positive sequences;

$c_{p}^{+}$- the subset of $c_{p}$ of decreasing sequences.

Let $\left\{\mu_{n}\right\} \in c$. Define $\left\{\mu_{n}^{+}\right\}$to be the sequence consisting of the terms of $\left\{\mu_{n}\right\}$ arranged in decreasing order. If $\left\{\mu_{n}\right\} \in c$, then $\left\{\mu_{n}^{(k)}\right\}$ is the sequence formed from $\left\{\mu_{n}\right\}$ by repeating each $\mu_{n}, k$ times.

Let $J \subset c$. We say that $J$ is an ideal set if it satisfies the following:

(1.1) If $\left\{\lambda_{n}\right\} \in J$, then $\left\{\lambda_{\pi(n)}\right\} \in J$ where $\pi$ is any permutation of the integers.

(1.2) If $\left\{\lambda_{n}\right\} \in J$ and if $0 \leqslant \mu_{n} \leqslant \lambda_{n}$, then $\left\{\mu_{n}\right\} \in J$.

(1.3) If $\left\{\mu_{n}\right\} \in J$ and $\left\{\lambda_{n}\right\} \in J$, then $\left\{\mu_{n}+\lambda_{n}\right\} \in J$.

For example, $c_{0}, c$, and $c_{p}$ are all ideal sets.

If $\mathcal{H C}$ is a separable Hilbert space and $J$ is an ideal set then there is a unique two-sided ideal, $\mathcal{G}$, of operators $T \in \mathscr{B}(\mathcal{H})$ whose sequence of singular numbers $\left\{s_{n}(T)\right\} \in J$. Conversely, to any ideal $g \subset \mathscr{B}(\mathcal{H})$ there corresponds in the same way a unique ideal set. For example, to the ideal of compact operators $\mathscr{K}(\mathcal{H})$ corresponds $c_{0}$, and to the ideal of Hilbert-Schmidt operators corresponds $c_{2}$.

If $J$ is an ideal set, then $J^{+}$, the subset of decreasing sequences, is called a characteristic set. Conversely, from any subset $J^{+}$of $c_{0}^{+}$satisfying (1.2) and (1.3) and the condition that $\lambda_{n}^{(2)} \in J^{+}$if $\left\{\lambda_{n}\right\} \in J^{+}$, we can generate an ideal set by including all permutations of all sequences in $J^{+}$. This result of Morris and Salinas [6] helps to facilitate the study of ideals. Indeed there is a one-to-one lattice isomorphism from the lattice of ideals of $\mathcal{K}(\mathcal{H})$ onto the lattice of characteristic sets. Thus any result established about characteristic sets yields an analogue for ideals of operators.

Let $T \in \mathscr{K}(\mathcal{F})$. We denote by $\mathcal{P}(T)$ the principal ideal generated by $T$ and by $P\left(\left\{s_{k}(T)\right\}\right)$ the characteristic set corresponding to $\mathscr{P}(T)$. Let $P\left(\left\{\lambda_{n}\right\}\right)$ be the characteristic set corresponding to the principal ideal of any positive 
operator $T$ with eigenvalues $\left\{\lambda_{n}\right\}$ counted according to multiplicity. Morris and Salinas [6] characterized such sets in the following theorem.

TheOREM A. Let $\left\{\lambda_{n}\right\} \in c_{0}^{+}$. Then

$$
P\left(\left\{\lambda_{n}\right\}\right)=\left\{\left\{\mu_{n}\right\} \in c_{0}^{+}: \mu_{n}=O\left(\lambda_{n}^{(k)}\right) \text {, for some integer } k\right\},
$$

or, equivalently,

$$
P\left(\left\{\lambda_{n}\right\}\right)=\left\{\left\{\mu_{n}\right\} \in c_{0}^{+}: \sup _{n} \mu_{n k} / \lambda_{n}<\infty, \text { for some integer } k\right\} .
$$

In association with $P\left(\left\{\lambda_{n}\right\}\right)$ we define

$$
\begin{gathered}
O\left(\left\{\lambda_{n}\right\}\right)=\left\{\left\{\mu_{n}\right\} \in c_{0}^{+}: \mu_{n}=O\left(\lambda_{n}\right)\right\}, \\
o\left(\left\{\lambda_{n}\right\}\right)=\left\{\left\{\mu_{n}\right\} \in c_{0}^{+}: \mu_{n}=o\left(\lambda_{n}\right)\right\} .
\end{gathered}
$$

Let $\left\{\mu_{n}\right\} \in c_{0}^{+}$and $\left\{m_{n}\right\}$ be a sequence of integers. We write $\left\{\mu_{n} ; m_{n}\right\}$ to denote the sequence

$$
\underbrace{\mu_{1}, \ldots, \mu_{1}}_{m_{1}}, \underbrace{\mu_{2}, \ldots, \mu_{2}}_{m_{2}}, \ldots, \underbrace{\mu_{n}, \ldots, \mu_{n}}_{m_{n}}, \ldots
$$

2. Regularity conditions. In this section we shall consider sequences in $c_{0}^{+}$ which satisfy various types of regularity conditions. Principal ideals generated by operators whose singular numbers satisfy these conditions will be seen to exhibit interesting properties and particularly simple structure. We begin with a rather weak type of regularity.

Definition 2.1. The sequence $\left\{\lambda_{n}\right\} \in c_{0}^{+}$is said to be semiregular if for every integer $k \geqslant 1$, we have $\sup _{n} \lambda_{n} / \lambda_{n k}=c_{k}<\infty$.

For example, the sequences $\left\{n^{-p}\right\}$, and $\left\{(\log n)^{-p}\right\}, p>0$, are semiregular, while $\left\{2^{-n}\right\}$ is not.

DEFINITION 2.2. The sequence $\left\{\lambda_{n}\right\} \in c_{0}^{+}$is said to be regular if $\sum_{j=1}^{n} \lambda_{j}=$ $O\left(n \lambda_{n}\right)$.

If the sequence $\left\{\lambda_{n}\right\} \in c_{0}^{+}$is regular then we have the inequalities

$$
\sum_{j=1}^{n k} \lambda_{j} \leqslant c n k \lambda_{n k}, \quad \sum_{j=1}^{n} \lambda_{j} \geqslant n \lambda_{n} .
$$

From

$$
1 \leqslant \sum_{j=1}^{n k} \lambda_{j} / \sum_{j=1}^{n} \lambda_{j} \leqslant \frac{c k \lambda_{n k}}{\lambda_{n}},
$$

we have

THEOREM 2.3. If the sequence $\left\{\lambda_{n}\right\} \in c_{0}^{+}$is regular then it is also semiregular.

In the following result we give an alternate characterization of regularity, which is a generalization of a result of Altshuler [1]. 
THEOREM 2.4. The sequence $\left\{\lambda_{n}\right\} \in c_{0}^{+}$is regular if and only if for some integer $k \geqslant 2, \sum_{j=1}^{k n} \lambda_{j} / \sum_{j=1}^{n} \lambda_{j} \geqslant l_{k}>1$ for all $n$.

Proof. It is sufficient to consider $k=2$. Suppose that $\left\{\lambda_{n}\right\}$ is regular. Then

$$
\sum_{j=1}^{2 n} \lambda_{j} / \sum_{j=1}^{n} \lambda_{j} \leqslant \frac{c 2 n \lambda_{2 n}}{n \lambda_{n}}
$$

and thus $\lambda_{2 n} / \lambda_{n} \geqslant(2 c)^{-1}$. So,

$$
\frac{\sum_{j=1}^{2 n} \lambda_{j}-\sum_{j=1}^{n} \lambda_{j}}{\sum_{j=1}^{n} \lambda_{j}} \geqslant \frac{n \lambda_{2 n}}{c n \lambda_{n}} \geqslant \frac{1}{2 c^{2}}
$$

and

$$
\sum_{j=1}^{2 n} \lambda_{j} / \sum_{j=1}^{n} \lambda_{j} \geqslant 1+\left(2 c^{2}\right)^{-1}
$$

On the other hand, if for some $k$ and all $n, \sum_{j=1}^{k n} \lambda_{j} / \sum_{j=1}^{n} \lambda_{j} \geqslant l_{k}>1$, then

$$
l_{k}-1 \leqslant \frac{\sum_{j=1}^{k n} \lambda_{j}-\sum_{j=1}^{n} \lambda_{j}}{\sum_{j=1}^{n} \lambda_{j}}<\frac{k n \lambda_{n}}{\sum_{j=1}^{n} \lambda_{j}} .
$$

Thus $\Sigma^{n} \lambda_{j} \leqslant k\left(l_{k}-1\right)^{-1} n \lambda_{n}$, and the theorem is proved.

The condition of regularity on the sequence $\left\{\lambda_{n}\right\}$ is a necessary and sufficient condition that the Lorentz sequence spaces generated by $\left\{\left\{\mu_{n}\right\} \in\right.$ $\left.c_{0}^{+}: \Sigma \lambda_{n} \mu_{n}^{p}<\infty\right\}, p>1$, be uniformly convex [1]. A slightly different type of regularity is contained in the following definition. The sequence $\left\{\lambda_{n}\right\} \in c_{0}^{+}$is said to be regularly decreasing if there exists an integer $k>2$ such that for each $n=1,2, \ldots$

$$
\lambda_{n} \leqslant \sum_{k(n-1)+1}^{k n} \lambda_{j}
$$

This definition was used by Salinas [8] in the study of commutants of ideals of operators. Certain subclasses of semiregular sequences are regularly decreasing. In particular, if $\left\{\lambda_{n}\right\} \in c_{0}^{+}$is semiregular with $c_{k} \leqslant k$ for some $k$ then

$$
\lambda_{n} \leqslant c_{k} \lambda_{n k} \leqslant \sum_{j=(n-1) k+1}^{n k} \lambda_{j}
$$

and so $\left\{\lambda_{n}\right\}$ is regularly decreasing. On the other hand, if $\left\{\lambda_{n}\right\}$ is regularly decreasing then it is obvious that $\left\{\lambda_{n}\right\}$ is semiregular.

That certain semiregular sequences are regular is the content of the next result.

THEOREM 2.6. Let $\left\{\lambda_{n}\right\} \in c_{0}^{+}$be a semiregular sequence for which 
$\sup _{n} \lambda_{n} / \lambda_{n k}=c_{k}<k$ for some $k$. Then $\left\{\lambda_{n}\right\}$ is regular.

Proof. From the inequality

$$
\lambda_{n} \leqslant c_{k} \lambda_{n k}<\frac{c_{k}}{k} \sum_{j=k(n-1)+1}^{k n} \lambda_{j}
$$

we have

$$
\sum_{j=1}^{n} \lambda_{j} \leqslant \frac{c_{k}}{k} \sum_{j=1}^{k n} \lambda_{j}
$$

Now since $c_{k} / k<1$, an application of Theorem 2.4 gives that $\left\{\lambda_{n}\right\}$ is regular.

We will conclude this section by considering aspects of a regular sequence as reflected by its frequency sequence.

Definition 2.7. For $c>1$, let $I_{j}(c)=\left[c^{-j}, c^{-j+1}\right)$. For any $\left\{\mu_{n}\right\} \in c_{0}^{+}$ define $M_{j}(\mu ; c)$ to be the number of $\mu_{n}^{\prime}$ 's in $I_{j}(c)$. We also define the cummulative frequency

$$
N_{n}(\mu ; c)=\sum_{j=1}^{n} M_{j}(\mu ; c)
$$

If $c=2$ we write $N_{n}(\mu)=N_{n}(\mu ; 2)$ and $M_{n}(\mu)=M_{n}(\mu ; 2)$.

With these notions we can give necessary and sufficient conditions that sequences be regular or semiregular.

THEOREM 2.8. Let $\left\{\mu_{n}\right\} \in c_{0}^{+}$, with $\mu_{1}<1$. A necessary and sufficient condition that $\left\{\mu_{n}\right\}$ be semiregular is that there exists $c, k>1$ such that $N_{n+1}(\mu ; c) / N_{n}(\mu ; c) \geqslant k$, for all $n$.

Proof. Suppose that $\left\{\mu_{n}\right\}$ is semiregular, and let $c=\sup \mu_{n} / \mu_{2 n}$. Suppose with no loss in generality that $\mu_{1} \geqslant 1 / c$; and so $N_{1}(\mu ; c) \neq 0$. If $j<N_{1}(\mu ; c)$ then $\mu_{2 j} \in I_{1}(c) \cup I_{2}(c)$. Thus $N_{2}(\mu ; c) \geqslant 2 N_{1}(\mu ; c)$. Similarly, if $j \leqslant N_{n}(\mu$; $c)$ then $\mu_{2 j} \in I_{1}(c) \cup \ldots \cup I_{n+1}(c)$. Thus $N_{n+1}(\mu ; c) \geqslant 2 N_{n}(\mu ; c)$, and the proof is complete with $k=2$.

Suppose now that $N_{n+1}(\mu ; c) / N_{n}(\mu ; c) \geqslant 2$ for all $n$. Reasoning as above we see that if $N_{n-1}(\mu ; c)<j \leqslant N_{n}(\mu ; c)$, then $2 j \leqslant N_{n+1}(\mu ; c)$ and $\mu_{j} / \mu_{2 j}<$ $c^{2}$. Thus if $k \geqslant 2$ the sequence $\left\{\mu_{j}\right\}$ is semiregular.

The proof will be complete if we establish that if $N_{n+1}(\mu ; c) / N_{n}(\mu$; $c) \geqslant k, 1<k<2$, and if $m=\left[(k-1)^{-1}\right]+1$, then $N_{n+1}\left(\mu ; c^{m}\right) / N_{n}(\mu$; $\left.c^{m}\right) \geqslant 2$. First observe that $N_{n}\left(\mu ; c^{m}\right)=N_{n m}(\mu ; c)$. So 


$$
\begin{aligned}
N_{n+1}\left(\mu ; c^{m}\right) / N_{n}\left(\mu ; c^{m}\right) & =N_{m(n+1)}(\mu ; c) / N_{m n}(\mu ; c) \\
& =\frac{N_{n m+1}(\mu ; c)}{N_{m n}(\mu ; c)}+\sum_{j=2}^{m} \frac{M_{m n+j}(\mu ; c)}{N_{n m}(\mu ; c)} \\
& >k+\sum_{j=2}^{m}(k-1)=m(k-1)+1 \\
& =\left(\left[(k-1)^{-1}\right]+1\right)(k-1)+1 \geqslant 2,
\end{aligned}
$$

and we are done.

From the proof of this theorem we obtain

COROLlARy 2.9. If $\left\{\mu_{n}\right\} \in c_{0}^{+}$is semiregular with $\mu_{1}<1$, and if $c$ and $k$ are as in the proof of Theorem 2.8, then $N_{n+1}(\mu ; c) \geqslant k^{n} N_{1}(\mu ; c)$.

In $\$ 4$ a special role will be played by subclasses of the semiregular sequences $\left\{\mu_{n}\right\}$ for which the ratios $\left\{N_{n}(\mu) / N_{n+1}(\mu)\right\} \in c_{p}^{+}$, for some $p \geqslant 1$. (Namely, we will show that for such a $\left\{\mu_{n}\right\}$, the sets $P\left(\left\{\mu_{n}\right\}\right)$ generate Orlicz spaces.) Such sequences descend very slowly. For example $\left\{(\log n)^{-1}\right\}$, $n \geqslant 3$, satisfies this condition, but the semiregular sequences $\left\{n^{-p}\right\}, p>0$, do not. Indeed, if $c=2$ and $\left\{\mu_{n}\right\}=\left\{(\log (n+2))^{-1}\right\}$, then $N_{n}(\mu) / N_{n+1}(\mu)$ $\sim e^{-2^{n}}$ which is summable for all $p$. But if $\left\{\lambda_{n}\right\}=\left\{\mu^{-q}\right\}$, then $N_{n}(\mu) / N_{n+1}(\mu) \sim 2^{-1 / q}$, and the sequence $N_{n}(\mu) / N_{n+1}(\mu)$ is not summable for any $p$. From this viewpoint these two sequences are vastly different.

CoRollaRY 2.10. If for some $c<\sqrt{2}, N_{n+1}(\mu ; c) / N_{n}(\mu ; c) \geqslant 2$, then $\left\{\mu_{n}\right\}$ is regular.

Proof. By hypothesis $\mu_{N_{k}(\mu ; c)} / \mu_{2 N_{k}(\mu ; c)}<c^{2}$. Now if $N_{k-1}(\mu ; c)<j \leqslant$ $N_{k}(\mu ; c)$, then

$$
\mu_{j} / \mu_{2 j}=\mu_{j} / \mu_{N_{k}} \cdot \mu_{N_{k}} / \mu_{2 j}<c^{2} \leqslant 2,
$$

and the result follows from Theorem 2.6.

3. Principal ideals and regularity conditions. In considering characteristic sets generated by regular or semiregular sequences, we shall discover they have a very simple structure. In fact our main result will establish that if the sequence is regular, principal ideals have a norm structure. The types of normed ideals in which we are interested in are contained in

Definition 3.1. Let $\left\{\lambda_{n}\right\} \in c_{0}^{+}$. Define $\mathcal{S}_{\Lambda}$ to be the set of compact operators $T$ whose singular numbers $\left\{s_{n}(T)\right\}$ satisfy

$$
\sup _{n} \sum_{j=1}^{n} s_{j}(T) / \sum_{j=1}^{n} \lambda_{j}<\infty .
$$

Define $\mathfrak{S}_{\Lambda}^{0}$ to be the subset of $\mathcal{S}_{\Lambda}$ for which 


$$
\lim _{m \rightarrow \infty} \sup _{n} \sum_{j=m}^{n+m} s_{j}(T) / \sum_{j=1}^{n} \lambda_{j}=0 .
$$

In Gohberg and Krein [5] it is shown that $\mathcal{S}_{\Lambda}^{0}$ and $\mathcal{S}_{\Lambda}$ are minimal and maximal ideals respectively with respect to the norm

$$
\|T\|_{\Lambda}=\sup _{n} \sum_{j=1}^{n} s_{j}(T) / \sum_{j=1}^{n} \lambda_{j}
$$

(We always assume that $\lambda_{1}=1$ to guarantee that the norm of rank one projections be 1.) If $\left\{\lambda_{n}\right\} \in c_{1}^{+}$then $\delta_{\Lambda}=\delta_{1}$, the ideal of nuclear operators. If $\left\{\lambda_{n}\right\} \in c_{0}^{+} \backslash c_{1}^{+}$, then $\mathfrak{S}_{\Lambda}^{0} \subsetneq \delta_{\Lambda}$ and $\delta_{\Lambda}$ is a nonseparable, nonreflexive Banach space.

Denote by $S_{\Lambda}^{0}$ and $S_{\Lambda}$ the characteristic sets corresponding to $\mathcal{S}_{\Lambda}^{0}$ and $\mathcal{S}_{\Lambda}$, respectively. We refer to the norm on $S_{\Lambda}^{0}$ and $S_{\Lambda}$ as that metric induced by the norm $\|\cdot\|_{\Lambda}$ on $\delta_{\Lambda}$. The following two theorems are proved in [5].

THEOREM B. Let $\left\{\lambda_{n}\right\}$ and $\left\{\pi_{n}\right\} \in c_{0}^{+}$. Then $S_{\Lambda}=S_{\Pi}$ if and only if

$$
\sup _{n} \sum_{j=1}^{n} \lambda_{j} / \sum_{j=1}^{n} \pi_{j}<\infty, \quad \sup _{n} \sum_{j=1}^{n} \pi_{j} / \sum_{j=1}^{n} \lambda_{j}<\infty .
$$

Theorem C. Let $\left\{\lambda_{j}\right\} \in c_{0}^{+}$. Then $S_{\Lambda}^{0}$ is characterized as the subset of $c_{0}^{+}$of sequences $\left\{\mu_{n}\right\}$ for which

$$
\lim _{n \rightarrow \infty} \sum_{j=1}^{n} \mu_{j} / \sum_{j=1}^{n} \lambda_{j}=0 .
$$

In this section we will show that $P\left(\left\{\lambda_{n}\right\}\right)=O\left(\left\{\lambda_{n}\right\}\right)$ if and only if $\left\{\lambda_{n}\right\}$ is semiregular, that $P\left(\left\{\lambda_{n}\right\}\right)=S_{\Lambda}$ if and only $\left\{\lambda_{n}\right\}$ is regular, that $S_{\Lambda}^{0}=o\left(\left\{\lambda_{n}\right\}\right)$ if and only if $\left\{\lambda_{n}\right\}$ is regular, that $S_{\Lambda}^{0} \subsetneq P\left(\left\{\lambda_{n}\right\}\right)$ if and only if $\lambda_{n}$ is not regular, and finally that $\mathcal{P}(T)$ is symmetrically normed if and only if $\left\{\lambda_{n}\right\}$ is regular.

Theorem 3.3. Let $\left\{\lambda_{n}\right\} \in c_{0}^{+}$. Then $P\left(\left\{\lambda_{n}\right\}\right)=O\left(\left\{\lambda_{n}\right\}\right)$ if and only if $\left\{\lambda_{n}\right\}$ is semiregular.

Proof. The condition of semiregularity on $\left\{\lambda_{n}\right\}$ clearly implies that $\left\{\lambda_{n}^{(k)}\right\}$ $\in O\left(\left\{\lambda_{n}\right\}\right)$, and hence that $P\left(\left\{\lambda_{n}\right\}\right)=O\left(\left\{\lambda_{n}\right\}\right)$ by Theorem A. Conversely if $\left\{\lambda_{n}^{(k)}\right\} \in P\left(\left\{\lambda_{n}\right\}\right)$, then $\lambda_{n}^{(k)}=O\left(\lambda_{n}\right)$ and, therefore, $\sup _{n} \lambda_{n} / \lambda_{n k}<\infty$, for each $k$.

So we see that if $\left\{\lambda_{n}\right\}$ is semiregular the principal characteristic set generated by $\left\{\lambda_{n}\right\}$ has a particularly simple structure. In the case that $\left\{\lambda_{n}\right\}$ is regular we have the following result.

TheOREM 3.4. Let $\left\{\lambda_{n}\right\} \in c_{0}^{+} \backslash c_{1}^{+}$. Then $P\left(\left\{\lambda_{n}\right\}\right)=S_{\Lambda}$ if and only if $\left\{\lambda_{n}\right\}$ is regular. 
To prove this result we will need several lemmas.

Lemma 3.5. If $\left\{\lambda_{n}\right\} \in c_{0}^{+}$then $P\left(\left\{\lambda_{n}^{(k)}\right\}\right)=P\left(\left\{\lambda_{n}\right\}\right)$, and $S_{\Lambda^{(k)}}=S_{\Lambda}$, for $k=1,2, \ldots$.

Proof. We know that $P\left(\left\{\lambda_{n}\right\}\right) \subseteq P\left(\left\{\lambda_{n}^{(k)}\right\}\right)$; the other inclusion $P\left(\left\{\lambda_{n}^{(k)}\right\}\right)$ $\subset P\left(\left\{\lambda_{n}\right\}\right)$ is obvious because $\left\{\lambda_{n}^{(k)}\right\} \in P\left(\left\{\lambda_{n}\right\}\right)$. From the fact that the sequence $\left\{\lambda_{n}^{(k)}\right\}$ is termwise greater than $\left\{\lambda_{n}\right\}$ we see that $S_{\Lambda^{(k)}} \supseteq S_{\Lambda}$. But

$$
\sum_{j=1}^{n k} \lambda_{j}^{(k)} / \sum_{j=1}^{n k} \lambda_{j}=k \sum_{j=1}^{n} \lambda_{j} / \sum_{j=1}^{n k} \lambda_{j} \leqslant k .
$$

From this it follows that

$$
\sup _{n} \sum_{j=1}^{n} \lambda_{j}^{(k)} / \sum_{j=1}^{n} \lambda_{j}<\infty,
$$

and from Theorem B this gives $S_{\Lambda^{(k)}} \subseteq S_{\Lambda}$.

LeMma 3.6. Let $\left\{\lambda_{n}\right\} \in c_{0}^{+} \backslash c_{1}^{+}$. Then $S_{\Lambda}=O\left(\left\{\lambda_{n}\right\}\right)$ if and only if $\left\{\lambda_{n}\right\}$ is regular.

Proof. The necessity is proved in Gohberg and Krein [5, p. 143]. To prove the sufficiency we suppose that $\left\{\lambda_{n}\right\}$ is not regular. Then there is a strictly increasing sequence of integers $\left\{k_{n}\right\}$ such that

$$
\sum_{j=1}^{k_{m}} \lambda_{j} \geqslant m k_{m} \lambda_{k_{m}}
$$

Define

$$
\begin{gathered}
\alpha_{1}=\cdots=\alpha_{k_{1}}=\left(\sum_{j=1}^{k} \lambda_{j}\right) / k_{1}, \\
\alpha_{k_{1}+1}=\cdots=\alpha_{k_{2}}=\left[\sum_{j=k_{1}+1}^{k_{2}} \lambda_{j}\right] /\left(k_{2}-k_{1}\right),
\end{gathered}
$$

etc. Taking $k_{0}=0$, we have

$$
\sum_{j=1}^{k_{n}} \alpha_{j}=\sum_{i=1}^{n} \sum_{j=k_{i-1}+1}^{k_{i}} \lambda_{j}=\sum_{j=1}^{k_{n}} \lambda_{j},
$$

and thus for all $n$,

$$
\sum_{j=1}^{n} \alpha_{j} \leqslant \sum_{j=1}^{n} \lambda_{j}
$$

So, no matter what the selection of integers $\left\{k_{n}\right\}$, the sequence $\left\{\alpha_{n}\right\} \in S_{\Lambda}$. Now we apply the fact that $\left\{\lambda_{n}\right\} \notin c_{1}^{+}$to recursively select a sequence $\left\{k_{m}\right\}$ to satisfy (3.7) and 


$$
\frac{\left(\sum_{j=k_{n-1}+1}^{k_{n}} \lambda_{j}\right) /\left(k_{n}-k_{n-1}\right)}{\left(\sum_{j=1}^{k_{n}} \lambda_{j}\right) / k_{n}} \geqslant \frac{1}{2} .
$$

From (3.7) and (3.8) we see that $\alpha_{k_{m}} / \lambda_{k_{m}}>m / 2$; thus $\left\{\alpha_{n}\right\} \notin O\left(\left\{\lambda_{n}\right\}\right)$ and the lemma is proved.

LeMma 3.9. If $\left\{\lambda_{n}\right\} \in c_{0}^{+}$, and if $\left\{\lambda_{n}\right\}$ is not regular, then $\left\{\lambda_{n}^{(k)}\right\}$ is not regular.

The proof is elementary. We pass on to the proof of Theorem 3.4. If $\left\{\lambda_{n}\right\}$ is regular then $\left\{\mu_{n}\right\} \in S_{\Lambda}$ implies $\left\{\mu_{n}\right\} \in O\left(\left\{\lambda_{n}\right\}\right)$ by Lemma 3.6. An application of Theorem 2.3 gives that $S_{\Lambda} \subseteq P\left(\left\{\lambda_{n}\right\}\right)$. Since $P\left(\left\{\lambda_{n}\right\}\right) \subseteq S_{\Lambda}$ for all sequences $\left\{\lambda_{n}\right\} \in c_{0}^{+}$, we have $S_{\Lambda}=P\left(\left\{\lambda_{n}\right\}\right)$.

Suppose now that $\left\{\lambda_{n}\right\}$ is not regular. We shall construct a sequence $\left\{\alpha_{n}\right\} \in c_{0}^{+}$such that $\left\{\alpha_{n}\right\} \in S_{\Lambda}$ but $\left\{\alpha_{n}\right\} \notin O\left(\left\{\lambda_{n}^{(k)}\right\}\right)$, for each $k=$ $1,2, \ldots$ By Lemmas 3.6 and 3.9 there are sequences $\left\{\alpha_{1, j}\right\},\left\{\alpha_{2, j}\right\}, \ldots$, which satisfy $\left\{\alpha_{k j}\right\} \in S_{\Lambda^{(k)}}$ and $\left\{\alpha_{k, j}\right\} \notin O\left(\left\{\lambda_{n}^{(k)}\right\}\right)$. Since $\lambda_{n}^{(k)} \geqslant \lambda_{n}$ and, by Lemma 3.5, $S_{\Lambda^{(k)}}=S_{\Lambda}$, we have that each sequence $\left\{\alpha_{k, j}\right\}$ is bounded in the norm of $S_{\Lambda}$. If $a_{k}$ denotes the norm of $\left\{\alpha_{k, j}\right\}$ and if we define

$$
\alpha_{j}=\sum_{k=1}^{\infty} \alpha_{k j} /\left(a_{k} 2^{k}\right), \quad j=1,2, \ldots,
$$

it is obvious that $\left\{\alpha_{j}\right\} \in S_{\Lambda}$. But, from (3.10), there is a constant $c_{k}$ such that $\alpha_{j}>c_{k} \alpha_{k j}$ for each $k$. So, we see that $\left\{\alpha_{j}\right\} \notin \cup_{k=1}^{\infty} O\left(\left\{\lambda_{n}^{(k)}\right\}\right)=P\left(\left\{\lambda_{n}\right\}\right)$, and the proof is complete.

There are a number of results connecting principal characteristic sets of one sequence to the characteristic sets $S_{\Lambda}$ of another sequence. We summarize these in the next theorem.

THEOREM 3.11. Let $\left\{\lambda_{n}\right\},\left\{\pi_{n}\right\} \in c_{0}^{+} \backslash c_{1}^{+}$.

(i) If $P\left(\left\{\lambda_{n}\right\}\right)=S_{\Pi}$, then $\left\{\lambda_{n}\right\}$ is regular and, hence, $S_{\Lambda}=S_{\Pi}$.

(ii) If $P\left(\left\{\lambda_{n}\right\}\right)=P\left(\left\{\pi_{n}\right\}\right)$ and if $\left\{\pi_{n}\right\}$ is regular, then $\left\{\lambda_{n}\right\}$ is regular.

(iii) Let $\lambda_{n}^{\prime}=\left(\sum_{j=1}^{n} \lambda_{j}\right) / n$. Then $S_{\Lambda}=S_{\Lambda^{\prime}}$ if and only if $\left\{\lambda_{n}^{\prime}\right\}$ is regular.

Proof. (i) From hypothesis $S_{\Pi} \subseteq S_{\Lambda}$. If $S_{\Pi}=S_{\Lambda}$ we are finished by Theorem 3.4. On the other hand, if $S_{\Pi} \subsetneq S_{\Lambda}$ then it follows that

$$
\sup _{n} \sum_{j=1}^{n} \pi_{j} / \sum_{j=1}^{n} \lambda_{j}<\infty, \sup _{n} \sum_{j=1}^{n} \lambda_{j} / \sum_{j=1}^{n} \pi_{j}=\infty,
$$

by Theorem B. Since $\left\{\lambda_{n}\right\} \in S_{\Pi}$ we have a contradiction, and the proof is complete.

(ii) If $\left\{\pi_{n}\right\}$ is regular the proof follows from an application of Theorems 3.4 and 3.11(i).

(iii) Suppose $\left\{\lambda_{n}^{\prime}\right\}$ is regular. From $\lambda_{n}^{\prime} \geqslant \lambda_{n}$, 


$$
\sum_{j=1}^{n} \lambda_{j}^{\prime} / \sum_{j=1}^{n} \lambda_{j} \leqslant c n \lambda_{n}^{\prime} / \sum_{j=1}^{n} \lambda_{j}=c,
$$

we see that $S_{\Lambda^{\prime}}=S_{\Lambda^{\prime}}$.

If $S_{\Lambda}=S_{\Lambda^{\prime}}$, then

$$
\sup \sum_{j=1}^{n} \lambda_{j}^{\prime} / \sum_{j=1}^{n} \lambda_{j}=c<\infty
$$

and so

$$
\sum_{j=1}^{n} \lambda_{j}^{\prime} \leqslant c \sum_{j=1}^{n} \lambda_{j}=c n \lambda_{n}^{\prime}
$$

We now consider the relation of $S_{\Lambda}^{0}$ to $P\left(\left\{\lambda_{n}\right\}\right)$ in the case $\left\{\lambda_{n}\right\}$ is not regular. We require the following technical lemma.

LEMMA 3.12. Let $\left\{\pi_{n}\right\} \in c_{0}^{+}$and $\left\{\lambda_{n}\right\} \in c_{0}^{+}$. Let us write $\left\{\lambda_{n}\right\}=\left\{\mu_{n} ; k_{n}\right\}$, where $\left\{\mu_{n}\right\} \in c_{0}^{+}$is a strictly decreasing sequence, and $\left\{k_{n}\right\}$ is a sequence of integers. Let $m_{n}=\sum_{i=1}^{n} k_{i}$. Then

$$
\sup _{n} \sum_{i=1}^{n} \lambda_{i} / \sum_{i=1}^{n} \pi_{i}=\sup _{n} \sum_{i=1}^{m_{n}} \lambda_{i} / \sum_{i=1}^{m_{n}} \pi_{i} \text {. }
$$

Proof. If we can establish that

$$
\sup _{m_{k}<n<m_{k+1}} \frac{\sum_{i=1}^{n} \lambda_{i}}{\sum_{i=1}^{n} \pi_{i}}=\max \left\{\frac{\sum_{i=1}^{m_{k}} \lambda_{i}}{\sum_{i=1}^{m_{k}} \pi_{i}}, \frac{\sum_{i=1}^{m_{k+1} \lambda_{i}}}{\sum_{i=1}^{m_{k}} \pi_{i}}\right\}
$$

we will be done. Let $p$ be the smallest integer in $m_{k}<p<m_{k+1}$ such that

$$
\sum_{i=1}^{p} \lambda_{i} / \sum_{i=1}^{p} \pi_{i}>\sum_{i=1}^{m_{k}} \lambda_{i} / \sum_{i=1}^{m_{k}} \pi_{i}
$$

If no such $p$ exists then (3.13) holds. By definition

$$
\sum_{i=1}^{p} \lambda_{i} / \sum_{i=1}^{p} \pi_{i}>\sum_{i=1}^{p-1} \lambda_{i} / \sum_{i=1}^{p-1} \pi_{i}
$$

It is easy to see that

$$
\sum_{i=1}^{p} \lambda_{i} / \sum_{i=1}^{p} \pi_{i} \leqslant\left(\sum_{i=1}^{p} \lambda_{i}+\lambda_{p}\right) /\left(\sum_{i=1}^{p} \pi_{i}+\pi_{p}\right) \leqslant \sum_{i=1}^{p+1} \lambda_{i} / \sum_{i=1}^{p+1} \pi_{i},
$$

since $\pi_{p+1} \leqslant \pi_{p}$ and $\lambda_{p}=\lambda_{p+1}=\lambda_{m_{k+1}}$. We can now conclude that (3.13) holds.

THEOREM 3.14. If $\left\{\pi_{n}\right\} \in c_{0}^{+} \backslash c_{1}^{+}$is not regular then

$$
S_{\pi}^{0} \& P\left(\left\{\pi_{n}\right\}\right) \text {. }
$$

Proof. Using the facts that $\left\{\pi_{n}\right\}$ is not regular and $\lim _{n \rightarrow \infty} \pi_{n}=0$, we are 
able to construct a sequence of integers increasing to infinity for which the following hold:

$$
\begin{gathered}
n_{j}>(j-1) n_{j-1} ; \\
\pi_{n_{j-1}}>j \pi_{n j} ; \\
\sum_{i=1}^{n_{j}} \pi_{i} /\left(n_{j} \pi_{n_{j}}\right) \geqslant j^{4} .
\end{gathered}
$$

Define the sequence $\left\{\xi_{n}\right\}$ by

$$
\xi_{n}=j \pi_{n_{j}}, \quad \text { if }(j-1) n_{j-1}<n \leqslant j n_{j} \text {. }
$$

From (3.17) it is evident that $\left\{\xi_{n}\right\} \in c_{0}^{+}$. Now for every integer $k$,

$$
\sup _{n} \xi_{k n} / \pi_{n} \geqslant \sup _{n_{j} j>k} \xi_{k n_{j}} / \pi_{n_{j}}=\sup _{n_{j} j>k} j \pi_{n_{j}} / \pi_{n_{j}}=\infty \text {. }
$$

Thus, by Theorem A, $\left\{\xi_{n}\right\} \notin P\left(\left\{\pi_{n}\right\}\right)$.

If we can show that $\left\{\xi_{n}\right\} \in S_{\Pi}^{0}$ the theorem will be proved. To this end it is sufficient, by Theorem $\mathrm{C}$, to show that

$$
\lim _{m \rightarrow \infty} \sum_{i=1}^{m} \xi_{i} / \sum_{i=1}^{m} \pi_{i}=0
$$

Define the (monotonically decreasing) sequence

$$
r_{m}=\sup _{n>m} \sum_{i=1}^{n} \xi_{i} / \sum_{i=1}^{n} \pi_{i} \text {. }
$$

Then

$$
\lim _{n \rightarrow \infty} r_{n}=\lim _{m \rightarrow \infty} r_{m n_{m}}
$$

and by Lemma 3.12,

$$
r_{m n_{m}}=\sup _{k n_{k}>m n_{m}} \sum_{i=1}^{k n_{k}} \xi_{i} / \sum_{i=1}^{k n_{k}} \pi_{i} .
$$

From (3.19) and (3.20) we need only show that the limit of the right-hand side of $(3.20)$ is zero.

Define

$$
A_{j}=\sum_{i=(j-1) n_{j-1}}^{j n_{j}} \xi_{i}=\left(j n_{j}-(j-1) n_{j-1}\right) j \pi_{n_{j}}
$$

and

$$
B_{j}=\sum_{i=1}^{j n_{j}} \pi_{j}
$$

Then a short computation, using (3.18), gives $A_{j} / B_{j}<j^{-2}$. So 


$$
r_{m n_{m}}=\sup _{k>m} \sum_{j=1}^{k} A_{j} / B_{k} \leqslant \sup _{k>m}\left(\sum_{j=1}^{k} B_{j} / j^{2}\right) / B_{k} \text {. }
$$

If for some $\varepsilon>0$ we choose $N$ large enough so that $\sum_{N+1}^{\infty} j^{-2}<\varepsilon / 4$, and $m$ large enough so that $\left(\sum_{j=1}^{N} B_{j} / j^{2}\right) / B_{k}<\varepsilon / 4$, for $k \geqslant m$ (since $\left\{\pi_{n}\right\} \in c_{0}^{+} \backslash$ $c_{1}^{+}$), then

$$
\begin{aligned}
\sup _{k>m}\left(\sum_{j=1}^{k} B_{j} / j^{2}\right) / B_{k} & =\sup _{k>m}\left[\left(\sum_{j=1}^{N} B_{j} / j^{2}\right) / B_{k}+\left(\sum_{j=N+1}^{k} B_{j} / j^{2}\right) / B_{k}\right] \\
& <\sup _{k>m}\left[\varepsilon / 4+\sum_{j=N+1}^{k}\left(B_{j} / B_{k}\right) j^{-2}\right] \\
& \leqslant \sup _{k>m}\left[\frac{\varepsilon}{4}+\sum_{j=N+1}^{k} j^{-2}\right]<\frac{\varepsilon}{2} .
\end{aligned}
$$

Since $\varepsilon$ is arbitrary the theorem is proved.

CoRollary 3.21. Let $\left\{\pi_{n}\right\} \in c_{0}^{+} \backslash c_{1}^{+}$. Then $S_{\Pi}^{0}=o\left(\left\{\pi_{n}\right\}\right)$ if and only if $\left\{\pi_{n}\right\}$ is regular.

Proof. Suppose $\left\{\pi_{n}\right\}$ is not regular. Since $o\left(\left\{\pi_{n}\right\}\right) \subset P\left(\left\{\pi_{n}\right\}\right)$ and, by Theorem 3.14, $P\left(\left\{\pi_{n}\right\}\right) \succeq S_{\Pi}^{0}$, then $S_{\Pi}^{0} \neq o\left(\left\{\pi_{n}\right\}\right)$. The converse is proved in Gohberg and Krein [5].

COROLLARY 3.22. $S_{\Pi}^{0} \subsetneq P\left(\left\{\pi_{n}\right\}\right)$ if and only if $\left\{\pi_{n}\right\}$ is regular (that is, $\left.P\left(\left\{\pi_{n}\right\}\right)=S_{\Pi}\right)$.

Let $g$ be an ideal of $\mathscr{B}(H)$ and let $\delta_{1}$ denote the nuclear operators on $H$. The Köthe dual of $J$ is defined as the ideal

$$
g^{\times}=\left\{T \in \mathscr{B}(H): S T \in \mathcal{S}_{1} \text { for all } S \in \mathcal{g}\right\} .
$$

From Salinas [7, Remark 4.7e], we have that the second Köthe dual of a principal ideal $\mathscr{P}\{T\}$ is given by $\delta_{\Pi}$, where $\left\{\pi_{n}\right\}=\left\{s_{n}(T)\right\}$.

THEOREM 3.23. A principal ideal $\mathcal{P}(T)$ is (symmetrically) normable if and only if $\left\{\pi_{n}\right\}=\left\{s_{n}(T)\right\}$ is regular.

Proof. Suppose $\mathscr{P}(T)$ is a normed ideal, and let $\Phi$ be the norm of $\mathscr{P}(T)$. Let $\delta_{\Phi}^{0}$ and $\delta_{\Phi}$ denote, respectively, the minimal and maximal normed ideals relative to $\Phi$. Applying Theorem 4.8 of Salinas [7] we have $\delta_{\Phi}^{0} \subsetneq \mathscr{P}(T) \subset \delta_{\Phi}$. From Theorem 4.3 and Remark 4.7c of Salinas [7] we have

$$
\delta_{\Phi}=\left(\delta_{\Phi}^{0}\right)^{\times \times} \subseteq \mathcal{P} \times \times(T)=\delta_{\Pi} \subset \delta_{\Phi}^{\times \times}=\delta_{\Phi},
$$

where $\Pi=\left\{\pi_{n}\right\}=\left\{s_{n}(T)\right\}$. But then $\delta_{\Phi}=\delta_{\Pi}$. By Salinas [7, Theorem 2.3] 
we have $\mathfrak{S}_{\Phi}^{0}=\mathfrak{S}_{\Pi}^{0}$. Thus $\mathfrak{S}_{\Pi}^{0} \subsetneq \mathscr{P}(T) \subseteq \mathcal{S}_{\Pi}$, and $\left\{s_{n}(T)\right\}$ is regular.

On the other hand, if $\left\{s_{n}(T)\right\}$ is regular, then, from Theorem 3.4, $\mathcal{P}(T)=$ $\mathcal{S}_{\Pi}$, where $\left\{\pi_{n}\right\}=\left\{s_{n}(T)\right\}$.

4. The frequency method. We return to the consideration of sequences described in the form $\left\{2^{-n} ; M_{n}\right\}$. Blass and Weiss [2] have shown that it is quite sufficient to consider only such sequences for the purpose of studying ideal sets. We shall also need the characteristic sets first introduced by Brown, Pearcy, and Salinas [3]. Let $\mathbb{Q}$ denote the set of positive valued functions $f$ defined on $[0, \infty)$ with $f(0)=0, f(t)>0$ if $t>0, f(t)$ is increasing, and $\lim _{t \rightarrow 0} f(t)=0, \lim _{t \rightarrow \infty} f(t)=\infty$. The set $\mathbb{Q}$ is called the admissible functions. For each $f \in \mathbb{Q}$ we can define two characteristic sets:

$$
\begin{aligned}
& S(f)=\left\{\left\{\mu_{n}\right\} \in c_{0}^{+} \mid \sum f\left(\alpha \mu_{n}\right)<\infty \text { for some } \alpha>0\right\}, \\
& D(f)=\left\{\left\{\mu_{n}\right\} \in c_{0}^{+} \mid \sum f\left(\alpha \mu_{n}\right)<\infty \text { for all } \alpha>0\right\} .
\end{aligned}
$$

Both sets are properly contained by $c_{0}^{+}$.

It is not necessary to consider all admissible functions. The subclass of $\mathbb{Q}$ consisting of those functions constant on each $\left\{I_{n}(2)\right\}$ is clearly a sufficiently broad class. In fact, if $f \in \mathbb{Q}$ and if we define $f(x)=f\left(2^{-n}\right)$ for $x \in I_{n}(2)$, then $S(\bar{f})=S(f)$.

The following result is due to Salinas [7, p. 234].

Theorem D. Let $f \in \mathbb{Q}$, then $\left\{\mu_{n}\right\} \in D(f)$ if and only if there is a monotonically increasing sequence $\left\{\xi_{n}\right\}$ with $\lim \xi_{n}=\infty$ such that $\left\{\xi_{n} \mu_{n}\right\} \in$ $D(f)$.

A refinement of Salinas' technique gives that we can assume $\left\{\xi_{n} \mu_{n}\right\} \in c_{0}^{+}$.

The fundamental theorem relating classes $S(f)$ and $P\left(\left\{\lambda_{n}\right\}\right)$ is due to A. Schweinsberg [10]:

Theorem E. Let $A=\left\{f \in \mathbb{Q} \mid\left\{\lambda_{n}\right\} \in S(f)\right\}$. Then

$$
P\left(\left\{\lambda_{n}\right\}\right)=\bigcap_{f \in A} S(f) .
$$

There are a number of applications of this theorem. For example, let $f_{1}, f_{2} \in A$ be noncommensurable in the sense that there are two sequences $\left\{\mu_{n}\right\}$ and $\left\{\lambda_{n}\right\}$ in $c_{0}^{+}$for which

$$
\begin{array}{ll}
\left\{\mu_{n}\right\} \in S\left(f_{1}\right), & \left\{\lambda_{n}\right\} \in S\left(f_{2}\right), \\
\left\{\mu_{n}\right\} \notin S\left(f_{2}\right), & \left\{\lambda_{n}\right\} \notin S\left(f_{1}\right) .
\end{array}
$$

Then it is obvious (cf. Brown, Pearcy, and Salinas [3]) from Theorem E that $P\left(\left\{\mu_{n}\right\}\right) \& P\left(\left\{\lambda_{n}\right\}\right)$ and $P\left(\left\{\lambda_{n}\right\}\right) \& P\left(\left\{\mu_{n}\right\}\right)$, and we have

THEOREM 4.1. The principal ideals are not well ordered by inclusion. 
This result adds to the long list of not well ordered by inclusion results for ideals. There is, between $D(f)$ and $S(f)$, another set,

$$
M(f)=\left\{\left\{\mu_{n}\right\} \in c_{0}^{+} \mid \sum f\left(\mu_{n}\right)<\infty\right\},
$$

which may not be a characteristic set but certainly has a more definitive structure. For certain sequences $\left\{\lambda_{n}\right\} \in c_{0}^{+}$we can show $P\left(\left\{\lambda_{n}\right\}\right) \subseteq M(f)$ if $\left\{\lambda_{n}\right\} \in M(f)$.

Definition 4.2. Let $\left\{\lambda_{n}\right\} \in c_{0}^{+}$. We say that $\left\{\mu_{n}\right\}$ satisfies condition (*) if for every scalar $\alpha>1$ there is a positive integer $k$ such that $\sup _{n} \mu_{k n} / \mu_{n} \leqslant \alpha^{-1}$. For example, the sequences $\left\{n^{-p}\right\},\left\{2^{-p n}\right\},\left\{(n !)^{-p}\right\}, p>0$, all satisfy (*), but slowly decreasing sequences such as $\left\{(\log n)^{-1}\right\}$ do not.

Theorem 4.3. Let $\left\{\lambda_{n}\right\} \in c_{0}^{+}$. Then $P\left(\left\{\lambda_{n}\right\}\right)=\bigcap_{f \in A} M(f)$ if and only if $\left\{\lambda_{n}\right\}$ satisfies (*). (Here $\mathrm{A}$ is defined in Theorem $\mathrm{E}$.)

Proof. If $\left\{\lambda_{n}\right\}$ satisfies (*) it is a routine calculation to show that $P\left(\left\{\lambda_{n}\right\}\right)$ $\subset M(f)$ for every $f \in A$. Then by Theorem $E$ the result follows.

Now we assume $P\left(\left\{\lambda_{n}\right\}\right) \subset M(f)$ for every $f \in A$. By our remarks above we can assume that $\left\{\lambda_{n}\right\}=\left\{2^{-n} ; M_{n}\right\}$ and $f$ is constant on each $\left\{I_{n}\right\}$. By Lemma 2.1 of Schweinsberg [10] we conclude that

$$
\sum_{j=1}^{n} M_{j+m} \leqslant c \sum_{j=1}^{n} M_{j}=c N_{n} .
$$

From the fact that $N_{n}$ is diverging to $\infty$ we can assume that $N_{n+m}<c N_{n}$ for all $n$. Thus there is an integer $k$ such that $k N_{n-1}>N_{n+m}$ and, hence, $k N_{n}>N_{n+m}$. Therefore

$$
\lambda_{k N_{n}} / \lambda_{N_{n}}<2^{-(n+m)} / 2^{-n}=2^{-m}
$$

If $N_{n-1}<j \leqslant N_{n}$ we have

$$
\lambda_{k j} / \lambda_{j} \leqslant \lambda_{k N_{n-1}} / \lambda_{N_{n}}<2^{-m}
$$

Since $m$ is arbitrary we see that $\left\{\lambda_{n}\right\}$ satisfies (*), and the theorem is proved.

Our next application of the "frequency method" is contained

THEOREM 4.4. Let $\left\{\lambda_{n}\right\},\left\{\mu_{n}\right\} \in c_{0}^{+}$. Then $\mu_{n}=O\left(\lambda_{n}\right)$ if and only if $N_{n}(\mu)$ $\leqslant N_{n+k}(\lambda)$ for some $k$ and all $n$.

PROOF. If $\mu_{n}=O\left(\lambda_{n}\right)$, then for some $k, \mu_{n} \leqslant 2^{k} \lambda_{n}$. Thus if $\mu_{j} \geqslant 2^{-n}$, then $\lambda_{j} \geqslant 2^{-(n+k)}$ and, hence, $N_{n}(\mu) \leqslant N_{n+k}(\lambda)$.

To prove the converse, first replace each of the given sequences by its equivalent frequency sequence: $\left\{\bar{\mu}_{n}\right\}=\left\{2^{-n} ; M_{n}(\mu)\right\}$ and $\left\{\bar{\lambda}_{n}\right\}=$ $\left\{2^{-n} ; M_{n}(\lambda)\right\}$. We want to show that if $N_{n}(\mu) \leqslant N_{n+k}(\lambda)$ for all $n$, then

$$
\bar{\mu}_{N_{n+k}}(\lambda) \leqslant 2^{k+1} \bar{\lambda}_{N_{n+k}(\lambda)}
$$


From the hypothesis we have $\mu_{N_{n+k}(\lambda)}<\mu_{N_{n}(\mu)}$. If $n=1$, (4.5) is apparent. We proceed indirectly assuming

$$
\bar{\mu}_{N_{n-j+k}(\lambda)}<2^{k+1} \bar{\lambda}_{N_{n-j+k}(\lambda)} \text { for } j=1,2, \ldots, n-1 .
$$

To establish (4.5) we consider four cases. In the two cases

(i) $N_{n-1}(\mu)<N_{n}(\mu), N_{n-1+k}(\lambda)=N_{n+k}(\lambda)$, and

(ii) $N_{n-1}(\mu)=N_{n}(\mu), N_{n-1+k}(\lambda)=N_{n+k}(\lambda)$,

(4.5) follows immediately from (4.6) with $j=1$. In the third case,

(iii) $N_{n-1}(\mu)<N_{n}(\mu), N_{n-1+k}(\lambda)<N_{n+k}(\lambda)$,

we see that $\bar{\mu}_{N_{n}(\mu)} \in I_{n}, \bar{\lambda}_{N_{n+k}(\lambda)} \in I_{n+k}$; and so (4.5) holds. Finally if

(iv) $N_{n-1}(\mu)=N_{n}(\mu), N_{n-1+k}(\lambda)<N_{n+k}(\lambda)$,

we must have $N_{n}(\mu)<N_{n+k}(\lambda)$, and so $\bar{\mu}_{N_{n+k}(\lambda)} \leqslant 2^{-n}$. This, together with $\bar{\lambda}_{N_{n+k}(\lambda)} \in I_{n+k}$, gives (4.5).

To complete the proof suppose $N_{n+k-1}(\lambda)<j \leqslant N_{n+k}(\lambda)$; then $j>$ $N_{n-1}(\mu)$ or $\bar{\mu}_{j}<2^{-n+1}$. Since $\bar{\lambda}_{j}=2^{-n-k}$ we have $\bar{\mu}_{j} / \bar{\lambda}_{j}^{n+k}=2^{k+1}$. Now we need only recall that $1 \leqslant \mu_{j} / \bar{\mu}_{j}<2$ and $1 \leqslant \lambda_{j} / \bar{\lambda}_{j}<2$ and the proof is complete.

In Theorem 4.1 we proved that principal ideals are not well ordered by inclusion. Suppose that $P\left(\left\{\mu_{n}\right\}\right) \subsetneq P\left(\left\{\lambda_{n}\right\}\right)$. Then can we "interpolate" these two characteristic sets by a characteristic set of the same type? That is, does there exist a sequence $\left\{\rho_{n}\right\} \in c_{0}^{+}$such that

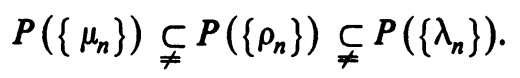

This question can be answered using the frequency method. We begin by proving some preliminary results which are interesting in themselves.

To establish (4.6) we will need to consider whether or nto $S(f) \backslash P\left(\left\{\mu_{n}\right\}\right)$ $\neq \varnothing$ whenever $\left\{\mu_{n}\right\} \in S(f)$. It turns out, surprisingly, that there are sequences $\left\{\mu_{n}\right\}$ and corresponding functions $f \in \mathbb{Q}$ such that

$$
P\left(\left\{\mu_{n}\right\}\right)=S(f) \text {. }
$$

The concept of semiregularity plays an important role in the ensuing discussion. We say that an admissible function $f \in \mathbb{Q}$ is monogenerating if there exist constants $\alpha>1, c>0$ and $\varepsilon>0$ such that $f(\alpha x)<c f(x)$ for $0<x<\varepsilon$.

THEOREM 4.8. (i) If $\left\{\mu_{n}\right\} \in c_{0}^{+}$is semiregular and $f \in \mathbb{Q}$ is a monogenerating function that sums $\left\{\mu_{n}\right\}$, then $P\left(\left\{\mu_{n}\right\}\right) \subsetneq S(f)$.

(ii) If $\left\{\mu_{n}\right\}$ is not semiregular and $f \in \mathbb{Q}$ sums $\left\{\mu_{n}\right\}$, then $P\left(\left\{\mu_{n}\right\}\right) \subsetneq S(f)$.

Proof. (i) Since $f$ is monogenerating, $D(f)=S(f)$ (cf. [3]) and, since $\left\{\mu_{n}\right\}$ is semiregular, $P\left(\left\{\mu_{n}\right\}\right)=O\left(\left\{\mu_{n}\right\}\right)$ by Theorem 3.3. From Theorem $\mathrm{D}$ there is an increasing sequence $\left\{\xi_{n}\right\}$ with limit $\infty$ such that $\left\{\xi_{n} \mu_{n}\right\} \in D(f)$. If 
$S(f)=P\left(\left\{\mu_{n}\right\}\right)$ then $\left\{\xi_{n} \mu_{n}\right\}=O\left(\mu_{n}\right)$. This is impossible and so $P\left(\left\{\mu_{n}\right\}\right) \subsetneq S(f)$.

(ii) We assume with no loss in generality that $\left\{\mu_{n}\right\} \in M(f)$. Then there exists an increasing sequence of integers $\left\{k_{n}\right\}$, with limit $\infty$, such that $\sum_{k=1}^{\infty} k_{n} f\left(\mu_{n}\right)<\infty$. Let $\left\{\xi_{n}\right\}=\left\{\mu_{n} ; k_{n}\right\}$. Clearly $\left\{\xi_{n}\right\} \in S(f)$. Now let $p_{n}=$ $\sum_{i=1}^{n} k_{i}$ and define $q_{n}$ by $p_{n}=q_{n} k+r_{n}, 0 \leqslant r_{n}<k$. It is clear that $\xi_{p_{n}}=\mu_{n}$ and $\mu_{p_{n}}^{(k)}=\mu_{q_{n}}$. Since $\lim p_{n} / n=\infty$, we see that $\lim q_{n} / n=\infty$. Now select $N$ such that $q_{n} / n \geqslant 2$ for all $n \geqslant N$. Then

$$
\sup _{n} \frac{\xi_{n}}{\mu_{n}^{(k)}} \geqslant \sup _{n} \frac{\xi_{p_{n}}}{\mu_{p_{n}}^{(k)}}=\sup _{n>N} \frac{\mu_{n}}{\mu_{q_{n}}} \geqslant \sup _{n>N} \frac{\mu_{n}}{\mu_{2 n}}=\infty,
$$

since $\left\{\mu_{n}\right\}$ is not semiregular, and the proof is complete.

From Theorem 4.8 we can deduce that if $P\left(\left\{\mu_{n}\right\}\right)=S(f)$ then (i) $\left\{\mu_{n}\right\}$ must be semiregular and (ii) $f \in \mathscr{Q}$ must not be monogenerating. In the following theorem we exhibit a class of sequences $\left\{\mu_{n}\right\}$ for which we can satisfy (4.7) for some $f \in \mathbb{Q}$.

THEOREM 4.9. Let $\left\{\mu_{n}\right\}=\left\{2^{-n}, M_{n}\right\}$ and suppose for some $p \geqslant 1$, $\left\{N_{n} / N_{n+1}\right\} \in c_{p}^{+}$. Then there exists an $f \in \mathbb{Q}$ such that $P\left(\left\{\mu_{n}\right\}\right)=S(f)$.

PROOF. If $\left\{\mu_{n}\right\}$ is as above we can assume with no loss in generality that $N_{n} / N_{n+1}<\frac{1}{2}$ and so $\left\{\mu_{n}\right\}$ is semiregular. Therefore $P\left(\left\{\mu_{n}\right\}\right)=O\left(\left\{\mu_{n}\right\}\right)$. If $\left\{\nu_{n}\right\} \notin O\left(\left\{\mu_{n}\right\}\right)$ then it is easy to see that $\sup _{n} \nu_{N_{n}} / \mu_{N_{n}}=\sup _{n} \nu_{N_{n}} / 2^{-n}=\infty$. Thus there is a sequence of integers $\left\{n_{k}\right\}$, increasing to $\infty$, such that $\nu_{N_{n k}} \geqslant 2^{-n} k^{k}$. Since $\left\{\nu_{n}\right\} \in c_{0}^{+}$we see that it is termwise greater than the sequence $\left\{\rho_{n}\right\}=\left\{2^{-n} k^{k} ; N_{n_{k}}-N_{n_{k-1}}\right\}$.

Now we define $f \in \mathscr{Q}$ to be constant on each $I_{n}$ and for which

$$
f\left(2^{-n}\right)=c_{n}^{p} / N_{n},
$$

where $c_{n}=N_{n} / N_{n+1}$. Then, by hypothesis,

$$
\sum_{n=1}^{\infty} f\left(\mu_{n}\right)=\sum_{n=1}^{\infty} M_{n} c_{n}^{p} / N_{n} \leqslant \sum c_{n}^{p}<\infty
$$

But

$$
\begin{aligned}
\sum_{n=1}^{\infty} f\left(\nu_{n}\right) & \geqslant \sum_{n=1}^{\infty} f\left(\rho_{n}\right)=\sum_{k=1}^{\infty}\left(N_{n_{k}}-N_{n_{k-1}}\right) c_{n_{k}-k}^{p} / N_{n_{k}-k} \\
& \geqslant c \sum_{k=1}^{\infty} N_{n_{k}} / N_{n_{k}-k} c_{n_{k}-k}^{p} \\
& \geqslant c \sum_{k=1}^{\infty}\left(\prod_{j=0}^{k-1} c_{n_{k}-k+j}^{-1}\right) c_{n_{k}-k}^{p}=\infty,
\end{aligned}
$$


since for all $k>p$ each term of the above series is greater than 1. This completes the proof.

It can be shown that the tails of the sequences defined in the hypothesis of Theorem 4.9 are regular. Moreover, it can be shown, using Corollary 2.9, that the functions $f$ constructed in the proof are essentially convex (but not mononormalizing). Thus there exist sequences $\left\{\pi_{n}\right\} \in c_{0}^{+}$for which we can satisfy the equality $P\left(\left\{\pi_{n}\right\}\right)=S(f)=S_{\Pi}$, where $S(f)$ generates an Orlicz space.

THEOREM 4.10. Let $\left\{\mu_{n}\right\}$ and $\left\{\lambda_{n}\right\} \in c_{0}^{+}$, and $P\left(\left\{\mu_{n}\right\}\right) \subsetneq P\left(\left\{\lambda_{n}\right\}\right)$. Then there exists a sequence $\left\{\rho_{n}\right\} \in c_{0}^{+}$for which (4.6)* holds.

Proof. With no loss in generality we can assume that $\mu_{n} \leqslant \lambda_{n}, n=$ $1,2, \ldots$ From Theorem $E$ there exists an $f \in \mathbb{Q}$ such that $\left\{\mu_{n}\right\} \in S(f)$ but $\left\{\lambda_{n}\right\} \notin S(f)$. Assume for the moment that $S(f) \backslash P\left(\left\{\mu_{n}\right\}\right) \neq \varnothing$. Then we can find a sequence $\left\{\rho_{n}\right\} \in S(f) \backslash P\left(\left\{\mu_{n}\right\}\right)$ which satisfies $\rho_{n} \leqslant \lambda_{n}$. Again by Theorem E, $P\left(\left\{\rho_{n}\right\}\right) \subsetneq P\left(\left\{\lambda_{n}\right\}\right)$ and, by construction, $P\left(\left\{\mu_{n}\right\}\right) \subsetneq P\left(\left\{\rho_{n}\right\}\right)$. This is (4.6)*, and the theorem is proved modulo the assumption.

In regard to the assumption $S(f) \backslash P\left(\left\{\mu_{n}\right\}\right) \neq \varnothing$ we see by Theorem 4.8 that the assumption is valid if $\left\{\mu_{n}\right\}$ is not semiregular. In the case $\left\{\mu_{n}\right\}$ is semiregular we have that $P\left(\left\{\mu_{n}\right\}\right)=O\left(\left\{\mu_{n}\right\}\right)$ by Theorem 3.3. Thus if

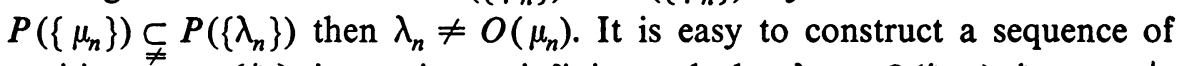
positive terms $\left\{\xi_{n}\right\}$, increasing to infinity such that $\lambda_{n} \neq O\left(\xi_{n} \mu_{n}\right), \xi_{n} \mu_{n} \in c_{0}^{+}$. Defining $\pi_{n}=\xi_{n} \mu_{n}$, it follows that $\pi_{n} \neq O\left(\mu_{n}\right)$, that $\pi_{n} \geqslant \lambda_{n}$, and that $\pi_{n} / \pi_{n k}$ $=\xi_{n} \mu_{n} /\left(\xi_{n k} \mu_{n k}\right) \leqslant \mu_{n} / \mu_{n k}<\infty$. This is to say that $\left\{\pi_{n}\right\}$ is semiregular and $P\left(\left\{\mu_{n}\right\}\right) \subset P\left(\left\{\pi_{n}\right\}\right)$. Since $\left\{\lambda_{n}\right\} \notin P\left(\left\{\pi_{n}\right\}\right)$ there is a function $f_{1} \in \mathbb{Q}$ such that $\left\{\lambda_{n}\right\} \notin S\left(f_{1}\right)$, and $\left\{\pi_{n}\right\} \in S\left(f_{1}\right)$. Since $S\left(f_{1}\right) \supseteq P\left(\left\{\pi_{n}\right\}\right)$ we have $S\left(f_{1}\right) \backslash$ $P\left(\left\{\mu_{n}\right\}\right) \neq \varnothing$. This proves the assumption was valid and the theorem is proved.

THEOREM 4.11. Let $\left\{\pi_{n}\right\},\left\{\lambda_{n}\right\} \in c_{0}^{+} \backslash c_{1}^{+}$satisfy

$$
\begin{gathered}
\pi_{n} \leqslant \lambda_{n}, \quad n=1,2, \ldots, \\
\sup _{n} \sum_{j=1}^{n} \lambda_{j} / \sum_{j=1}^{n} \pi_{j}=\infty .
\end{gathered}
$$

Then $P\left(\left\{\pi_{n}\right\}\right) \underset{\not}{ } P\left(\left\{\lambda_{n}\right\}\right)$.

Proof. From (4.12), $P\left(\left\{\pi_{n}\right\}\right) \subseteq P\left(\left\{\lambda_{n}\right\}\right)$. If $\left\{\lambda_{n}\right\} \in P\left(\left\{\pi_{n}\right\}\right)$, then $\left\{\lambda_{n}\right\} \in$ $S_{\Pi}$. But then, by Theorem C,

$$
\sup _{n} \sum_{j=1}^{n} \lambda_{j} / \sum_{j=1}^{n} \pi_{j}<\infty,
$$

which contradicts (4.13). 


\section{REFERENCES}

1. Z. Altshuler, Uniform convexity in Lorentz sequence spaces, Israel J. Math. 20 (1975), 260-274. MR 52 \#6378.

2. A. Blass and G. Weiss, $A$ characterization and sum decomposition for operator ideals (preprint).

3. A. Brown, C. Pearcy and N. Salinas, Ideals of compact operators on Hilbert space, Michigan Math. J. 18 (1971), 373-384. MR 45 \#909.

4. J. W. Calkin, Two-sided ideals and congruences in the ring of bounded operators in Hilbert space, Ann. of Math. (2) 42 (1941), 839-873. MR 3, 208.

5. I. C. Gohberg and M. G. Krein, Introduction to the theory of linear nonselfadjoint operators in Hilbert space, Transl. Math. Monographs, vol. 18, Amer. Math. Soc., Providence, R. I., 1969. MR 39 \# 7447.

6. D. Morris and N. Salinas, Semiprime ideals and irreducible ideals in the ring of bounded operators on Hilbert space, Indiana Univ. Math. J. 23 (1974), 575-589.

7. N. Salinas, Symmetric norm ideals and relative conjugate ideals, Trans. Amer. Math. Soc. 188 (1974), 213-240. MR 49 \# 1146.

8. _ Ideals of commutators of compact operators, Acta Sci. Math. 36 (1974), 130-144. MR 50 \# 10891.

9. Ideal sets and ideals of compact operators on Hilbert space, Indiana Univ. Math. J. 22 (1972/73), 505-521. MR 47 \#5642.

10. A. Schweinsberg, Principal ideals of compact operators, Indiana Univ. Math. J. 25 (1976), 229-234.

11. G. Weiss, Commutators and operator ideals, Ph.D. Thesis, 1975.

Department of Mathematics, Texas A\&M University, College Station, Texas 77843 